\title{
Weak hyperbolicity and free constructions
}

\author{
D.V. Osin
}

\begin{abstract}
The aim of this note is to show that weak relative hyperbolicity of a group relative to a subgroup (or relative hyperbolicity in the sense of Farb) does not imply any natural analogues of some well-known algebraic properties of ordinary hyperbolic groups. Our main tools are combination theorems for weakly relatively hyperbolic groups.
\end{abstract}

\section{Introduction}

Given a group $G$ generated by a set $S$, the Cayley graph $\Gamma=\Gamma_{S}(G)$ of $G$ is an oriented labelled 1-complex with the vertex set $G$ and the edge set $G \times S$. An edge $e=(g, s) \in E(\Gamma)$ goes from the vertex $g$ to the vertex $g s$ and has the label $\phi(e)=s$. The graph $\Gamma$ can be regarded as a metric space if we endow it with a combinatorial metric. This means that the length of every edge of $\Gamma$ is assumed to be equal to 1 .

Recall that a geodesic metric space $M$ is hyperbolic, if there exists $\delta \geq 0$ such that for any geodesic triangle $\Delta$ in $M$, every side of $\Delta$ is contained in the closed $\delta$-neighborhood of the union of the other two sides. A group $G$ is called hyperbolic if $G$ is generated by a finite set $X$ and the Cayley graph $\Gamma_{X}(G)$ is a hyperbolic metric space [13. This definition is independent of the choice of the finite generating set $X$. One can generalize the notion of a hyperbolic group as follows.

Definition 1.1. Let $G$ be a group, $\mathcal{H}=\left\{H_{1}, \ldots, H_{m}\right\}$ a collection of subgroups of $G$. A subset $X \subset G$ is a relative generating set of $G$ with respect to $\mathcal{H}$, if $G$ is generated by $X \cup H_{1} \ldots \cup H_{m}$. By the relative Cayley graph $\Gamma^{r e l}=\Gamma_{X}^{r e l}(G)$ of $G$ with respect to $\mathcal{H}$, we mean the Cayley graph of $G$ with respect to the generating set $X \cup H_{1} \cup \ldots \cup H_{m}$. We say that $G$ is weakly hyperbolic relative to $\mathcal{H}$, if there exists a finite relative generating set $X$ of $G$ with respect to $\mathcal{H}$ and the corresponding relative Cayley graph is hyperbolic.

2000 Mathematics Subject Classification. Primary 20F65; Secondary 20F67, 05C25, 20E06.

Key words and phrases. Relatively hyperbolic group, HNN-extension, free product with amalgamation, finitely presented group, word problem, simple group.

This work has been supported by the RFFR Grants $\sharp 02-01-00892, \sharp 03-01-06555$.

(C)0000 (copyright holder) 
It is straightforward to check that if $Y$ is another finite relative generating set of $G$, then the corresponding relative Cayley graphs $\Gamma_{X}^{r e l}(G)$ and $\Gamma_{Y}^{r e l}(G)$ are quasiisometric. Since hyperbolicity is preserved under quasi-isometries, our definition is independent of the choice of finite relative generating sets of $G$ with respect to $\mathcal{H}$. In case the groups $G, H_{1}, \ldots, H_{m}$ are finitely generated in the usual sense, Definition [1.1] is equivalent to the definition of relative hyperbolicity given by Farb 10 (see Lemma 2.4). Thus Definition 1.1 can be regarded as a generalization of Farb's one. We use the term 'weakly relatively hyperbolic group' to distinguish the class of groups considered in this paper from the class of relatively hyperbolic groups introduced by Bowditch in $\mathbf{3}$ (the last class is strictly larger, see 24). It should be noted that Theorems 1.2 1.3 stated below remain true if we replace the words 'weakly relatively hyperbolic' with 'relatively hyperbolic in the sense of Farb' and, in addition, require all groups and subgroups under consideration to be finitely generated.

It is known that if $G$ is hyperbolic with respect to a collection of subgroups $\left\{H_{1}, \ldots, H_{m}\right\}$ in the sense of Bowditch, then $G$ inherits some important algebraic and algorithmic properties of $H_{1}, \ldots, H_{m}$ such as finite presentability, decidability of various algorithmic problems, etc. [7, 10, 19, 21. The main purpose of this note is to show that this is not so in case of weak relative hyperbolicity. To construct the corresponding examples we use the following 'combination theorems' for amalgamated products and HNN-extensions. Other results of this type for hyperbolic and relatively hyperbolic groups can be found in 2, 9, 12, 14, 17.

TheOREm 1.2. Let $H$ be an arbitrary group, $A$ and $B$ two isomorphic subgroups of $H$. Denote by $G$ the $H N N$-extension of $H$ with associated subgroups $A$ and $B$.

(1) $G$ is weakly hyperbolic relative to $H$.

(2) If $H$ is weakly hyperbolic relative to $\{A, B\}$, then $G$ is weakly hyperbolic relative to $A$.

TheOrem 1.3. Let $H, K$ be arbitrary groups, $A$ and $B$ isomorphic subgroups of $H$ and $K$ respectively. Denote by $G$ the amalgamated free product $H *_{A=B} K$.

(1) $G$ is weakly hyperbolic relative to $\{H, K\}$.

(2) If $H$ is weakly hyperbolic relative to $A$ and $K$ is weakly hyperbolic relative to $B$, then $G$ is weakly hyperbolic relative to $A$.

We notice that if $U \leq V \leq W$ are groups such that $W$ is weakly hyperbolic relative to $V$ and $V$ is weakly hyperbolic relative to $U$, then, in general, $W$ is not weakly hyperbolic relative to $U$. For example, this is so for $U=\mathbb{Z}, V=U \times \mathbb{Z}$, $W=V \times \mathbb{Z}$. Thus the second assertion in each of these theorems can not be derived from the first ones.

In what follows we call a finitely generated group $G$ metahyperbolic, if $G$ is finitely generated and weakly hyperbolic relative to a hyperbolic subgroup $H \leq G$ (or, equivalently, $G$ is hyperbolic relative to a hyperbolic subgroup $H \leq G$ in the sense of Farb). Such groups are simplest non-trivial examples of relatively hyperbolic ones. Thus it would be reasonable to suspect that they are very close to ordinary hyperbolic groups from the algebraic point of view. However this is not so as the following examples show. 
Recall that any hyperbolic group is finitely presented [13. Generalizing this fact, the authors of [5] stated that if a finitely generated group $G$ is weakly hyperbolic relative to a finitely generated subgroup $H$, then $G$ is finitely presented with respect to $H$, which means that a presentation of $G$ can be obtained from a presentation of $H$ by adding a finite number of generators and relations. In particular, this would imply that any metahyperbolic groups is finitely presented in the usual sense. However, the proof contains a gap which arises from the absence of the local finiteness of the relative Cayley graph. The following corollary provides a counterexample.

COROLlaRY 1.4. There exists a metahyperbolic group which is not finitely presented.

It is well known that any hyperbolic group possess a finite presentation with Dehn property [16. In particular, the word problem is decidable for any hyperbolic group in linear time. Moreover, if $G$ is hyperbolic with respect to a subgroup $H$ and the word problem is decidable in $H$, then it is decidable in $G$ [10. (For other algorithmic problems in relatively hyperbolic groups we refer to [7, [19], and [21]). The next result shows that this can can not be generalized to the weak case.

COROLLARY 1.5. There exists a finitely presented metahyperbolic group with undecidable word problem.

The last corollary is inspired by the following result. If $G$ is an infinite hyperbolic group, then $G$ is never simple. Moreover, if $G$ is not cyclic-by-finite, then it contains uncountably many normal subgroups $[\mathbf{1 3}, \mathbf{1 8}$. The same is true in case $G$ is relatively hyperbolic in the sense of Bowditch with respect to an infinite proper subgroup $H$ [20].

COROLLARY 1.6. There exists a finitely presented infinite metahyperbolic simple group.

Acknowledgements. The author is grateful to Mike Mihalik and the referee for useful remarks concerning this paper.

\section{Equivalent definitions of weakly relatively hyperbolic groups}

We begin with various definitions of weak relative hyperbolicity. Throughout this section we fix a group $G$, a collection of subgroups $\mathcal{H}=\left\{H_{1}, \ldots, H_{m}\right\}$ of $G$, and a finite relative generating set $X$ of $G$ with respect to $\mathcal{H}$. For a graph $\Xi$, we denote by $V(\Xi)$ and $E(\Xi)$ the sets of vertices and edges of $\Xi$. If $e$ is an edge of $\Xi$, we write $e_{-}$and $e_{+}$for the origin and the terminus of $e$ respectively.

Definition 2.1. By the left coset graph $\widetilde{\Gamma}=\widetilde{\Gamma}_{X}(G)$ of $G$ with respect to $\mathcal{H}$ we mean the oriented labelled 1-complex constructed as follows. The vertex set of $\widetilde{\Gamma}$ is $V(\widetilde{\Gamma})=\left\{g H_{i}, i=1, \ldots, m, g \in G\right\}$. For two different cosets $f H_{i}$ and $g H_{j}$, there exists an (oriented) edge $e$ going from $f H_{i}$ to $g H_{j}$ if and only if there are elements $a \in f H_{i}$ and $b \in g H_{j}$ such that $b=a x$ for some $x \in X \cup X^{-1} \cup\{1\}$. The triple $(i, j, x)$ is called the label of $e$. Obviously $\widetilde{\Gamma}$ is connected and, in general, not locally finite.

The next definition was formulated by Farb $\mathbf{1 0}$. 
Definition 2.2. Suppose that the group $G$ is generated by the set $X$ in the usual (non-relative) sense. We begin with the Cayley graph $\Gamma_{X}(G)$ of $G$ and form a new graph as follows: for each left coset $g H_{i}, i=1, \ldots, m$, of $H_{i}$ in $G$, add a vertex $v\left(g H_{i}\right)$ to $\Gamma_{X}$, and add an edge $e(g h)$ of length $1 / 2$ from each element $g h$ of $g H_{i}$ to the vertex $v\left(g H_{i}\right)$. The new graph is called the coned-off Cayley graph of $G$ with respect to $\mathcal{H}$, and is denoted by $\widehat{\Gamma}=\widehat{\Gamma}_{X}(G)$.

We equip the graphs $\widetilde{\Gamma}$ and $\widehat{\Gamma}$ with combinatorial metrics. In case $G$ is finitely generated and $\widehat{\Gamma}$ is hyperbolic, the group $G$ is called hyperbolic relative to $\mathcal{H}$ in the sense of Farb $\mathbf{1 0}$.

Definition 2.3. Two metric spaces $M_{1}, M_{2}$ are said to be quasi-isometric if there exist $\lambda>0, c \geq 0, \varepsilon \geq 0$, and a map $\alpha: M_{1} \rightarrow M_{2}$ such that the following two condition hold.

(a) For any $x, y \in M_{1}$, we have

$$
\frac{1}{\lambda} \operatorname{dist}_{M_{1}}(x, y)-c \leq \operatorname{dist}_{M_{2}}(\alpha(x), \alpha(y)) \leq \lambda \operatorname{dist}_{M_{1}}(x, y)+c .
$$

(b) For any $z \in M_{2}$ there exists $x \in M_{1}$ such that

$$
\operatorname{dist}_{M_{2}}(\alpha(x), z) \leq \varepsilon
$$

The lemma below shows, in particular, that we can regard Definition 1.1 as a generalization of Farb's one. Recall that $\Gamma^{r e l}$ denotes the relative Cayley graph of $G$ with respect to $\mathcal{H}$ defined in the introduction.

LEMMA 2.4. The following conditions are equivalent.

(i) The graph $\Gamma^{\text {rel }}$ is hyperbolic.

(ii) The graph $\widetilde{\Gamma}$ is hyperbolic.

In case $G$ is generated by the set $X$ in the usual (non-relative) sense, the above conditions are equivalent to

(iii) The graph $\widehat{\Gamma}$ is hyperbolic.

Proof. (i) $\Leftrightarrow$ (ii). Recall that hyperbolicity (or the absence of it) is preserved when we pass from a metric space to a quasi-isometric one. We define a map $\alpha: V\left(\Gamma^{r e l}\right) \rightarrow V(\widetilde{\Gamma})$ by the rule $\alpha(g)=g H_{1}$ for any $g \in G$. Since any graph is quasi-isometric to its vertex set equipped with the induced metric, it suffices to show that $\alpha$ satisfies conditions (a) and (b) from Definition 2.3 for some $\lambda, c, \varepsilon$.

Suppose that two vertices $u, v$ are connected by an edge in $\Gamma^{r e l}$. Then there are only three possibilities. First assume that there exists $x \in X \cup X^{-1}$ such that $u=v x$. Clearly $\alpha(u)=u H_{1}$ and $\alpha(v)=v H_{1}$ are connected by an edge in $\widetilde{\Gamma}$ in this case. Next suppose $u H_{1}=v H_{1}$. Then $\alpha(u)=\alpha(v)$. Finally let $u H_{i}=v H_{i}$ for some $i=2, \ldots, m$. Then $\alpha(u)=u H_{1}$ is connected to $u H_{i}=v H_{i}$ in $\widetilde{\Gamma}$ by the edge labelled $(1, i, 1)$ and $v H_{i}$ is connected to $\alpha(v)=v H_{1}$ by the edge labelled $(i, 1,1)$.

Thus in all cases we have $\operatorname{dist}_{\widetilde{\Gamma}}(\alpha(u), \alpha(v)) \leq 2$. Obviously this implies

$$
\operatorname{dist}_{\widetilde{\Gamma}}(\alpha(u), \alpha(v)) \leq 2 \operatorname{dist}_{\Gamma^{r e l}}(u, v)
$$

for arbitrary $u, v \in \Gamma^{r e l}$. Further, if for some $u, v \in \Gamma^{r e l}, \alpha(u)$ and $\alpha(v)$ are connected by a path of length $n$ in $\widetilde{\Gamma}$, then $v=u h_{1} x_{1} h_{2} x_{2} \ldots h_{n} x_{n} h_{n+1}$, where 
$h_{1}, h_{n+1} \in H_{1}, h_{2}, \ldots, h_{n} \in \bigcup_{i=1}^{m} H_{i}$, and $x_{1}, \ldots, x_{n} \in X \cup X^{-1} \cup\{1\}$. Therefore, $\operatorname{dist}_{\Gamma^{r e l}}(u, v) \leq 2 n+1$, which yields

$$
\frac{1}{2} \operatorname{dist}_{\Gamma^{r e l}}(u, v)-\frac{1}{2} \leq \operatorname{dist}_{\widetilde{\Gamma}}(\alpha(u), \alpha(v))
$$

for any $u, v \in \Gamma^{r e l}$. Inequalities (10) and (2) together imply the first condition in Definition 2.3 for $\lambda=c=1 / 2$. It remains to notice that the second condition holds for $\varepsilon=1$ since any coset $u H_{i}$ in $\widetilde{\Gamma}$ is a distance of at most 1 from $u H_{1}=\alpha(u)$.

(ii) $\Leftrightarrow$ (iii). Note that the identity map on $G$ induces an isometric embedding $\iota$ of the vertex set $V\left(\Gamma^{r e l}\right)$ of $\Gamma^{r e l}$ to $\widehat{\Gamma}$ and $\widehat{\Gamma}$ belongs to the closed 1-neighborhood of the image $\iota\left(V\left(\Gamma^{r e l}\right)\right)$.

In the next section we will also use the following result.

LEMma 2.5. The group $G$ acts on $\widetilde{\Gamma}$ by left multiplications isometrically with a finite number of orbits of edges.

Proof. The fact that the action of $G$ is isometric is obvious. Let us prove that the number of orbits of edges is finite. Note that $G$ can act on $\widetilde{\Gamma}$ with inversions, so we can not speak about the quotient of $\widetilde{\Gamma}$ with respect to the action.

Clearly it suffices to show that any two edges having equal labels belong to the same orbit. Since the number of different labels is finite, this will imply the statement of the lemma. Let $f_{1} H_{i}, f_{2} H_{i}, g_{1} H_{j}, g_{2} H_{j}$ be cosets and $a_{1} \in f_{1} H_{i}$, $a_{2} \in f_{2} H_{i}, b_{1} \in g_{1} H_{j}, b_{2} \in g_{2} H_{j}$ elements of $G$ such that $b_{1}=a_{1} x$ and $b_{2}=a_{2} x$ for some $x \in X \cup X^{-1} \cup\{1\}$. We have to show that there exists $w \in G$ which takes the pair $\left(f_{1} H_{i}, g_{1} H_{j}\right)$ to $\left(f_{2} H_{i}, g_{2} H_{j}\right)$. Let $w$ be the element $a_{2} a_{1}^{-1}$. Obviously $w=f_{2} h f_{1}^{-1}$ for some $h \in H_{i}$. Thus $w$ takes $f_{1} H_{i}$ to $f_{2} H_{i}$. Further, note that

$$
w=a_{2} a_{1}^{-1}=b_{2} x x^{-1} b_{1}^{-1}=b_{2} b_{1}^{-1}=g_{2} k g_{1}^{-1}
$$

for some $k \in H_{j}$. Therefore, $w g_{1} H_{j}=g_{2} k H_{j}=g_{2} H_{j}$. This completes the proof.

\section{Proofs of the main results}

We start with auxiliary lemmas. The following is a version of Svarč-Milnor Lemma for non-proper actions of groups on (not necessary locally finite) graphs.

Lemma 3.1. Let $G$ be a group acting on graphs $\Gamma_{1}$ and $\Gamma_{1}$ with finite number of orbits of edges. Suppose that there is a bijection between the vertex sets $\beta: V\left(\Gamma_{1}\right) \rightarrow$ $V\left(\Gamma_{2}\right)$ such that the diagram

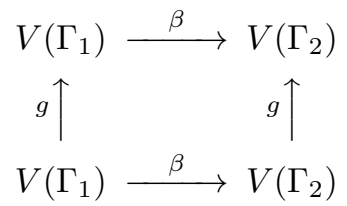

is commutative for any $g \in G$. Then the graphs $\Gamma_{1}$ and $\Gamma_{2}$ are quasi-isometric. 
Proof. Suppose that two edges $e, f \in E\left(\Gamma_{1}\right)$ belong to the same orbit, i.e., $g e=f$ for some $g \in G$. Let $p$ be a geodesic paths connecting $\beta\left(e_{-}\right)$to $\beta\left(e_{+}\right)$in $\Gamma_{2}$. Obviously $g p$ is a path of the same length as $p$ connecting $\beta\left(f_{-}\right)$to $\beta\left(f_{+}\right)$. Thus we have $\operatorname{dist}_{\Gamma_{2}}\left(\beta\left(e_{-}\right), \beta\left(e_{+}\right)\right)=\operatorname{dist}_{\Gamma_{2}}\left(\beta\left(f_{-}\right), \beta\left(f_{+}\right)\right)$. Since the number of orbits of edges is finite, there exists the maximum

$$
M=\max _{e \in E\left(\Gamma_{1}\right)} \operatorname{dist}_{\Gamma_{2}}\left(\beta\left(e_{-}\right), \beta\left(e_{+}\right)\right) .
$$

Thus for any $u, v \in V\left(\Gamma_{1}\right)$, we have

$$
\operatorname{dist}_{\Gamma_{2}}(\beta(u), \beta(v)) \leq M \operatorname{dist}_{\Gamma_{1}}(u, v) .
$$

The converse inequality can be obtained in the same way. Therefore, $\beta$ defines a quasi-isometry between vertex sets of $\Gamma_{1}$ and $\Gamma_{2}$, which yields the assertion of the lemma.

Let $\Sigma$ be a graph. For a cycle $c$ in $\Sigma$, we denote by $[c]$ its homology class in $H_{2}(\Sigma, \mathbb{Z})$. By $l(c)$ and $d(c)$ we denote the length and the diameter of $c$ respectively. The next proposition is a homological variant of the characterization of hyperbolic graphs by linear isoperimetric inequality (see [4, 6]).

Proposition 3.2. For any graph $\Sigma$ the following conditions are equivalent.

(i) $\Sigma$ is hyperbolic.

(ii) There are some positive constants $M, L$ such that if $c$ is a cycle in $\Sigma$, then there exist cycles $c_{1}, \ldots, c_{k}$ in $\Sigma$ with $d\left(c_{i}\right) \leq M$ for all $i=1, \ldots, k$ such that

$$
[c]=\left[c_{1}\right]+\ldots+\left[c_{k}\right]
$$

and $k \leq \operatorname{Ll}(c)$.

Lemma 3.3. Let $G$ be a group, $R$ a retract of $G$. Suppose that $G$ is weakly hyperbolic relative to a collection of subgroups $\mathcal{A}=\left\{A_{1}, \ldots, A_{m}\right\}$ and $A_{i} \leq R$ for all $i=i, \ldots, m$. Then $R$ is weakly hyperbolic relative to $\mathcal{A}$.

Proof. Let $X$ be a finite relative generating set of $G$ with respect to $\mathcal{A}, Y$ the image of $X$ under the retraction $G \rightarrow R$. Then the relative Cayley graph $\Gamma_{Y}^{r e l}(R)$ of $R$ with respect $\mathcal{A}$ is a retract of the relative Cayley graph $\Gamma_{X}^{r e l}(G)$ of $G$ with respect to $\mathcal{A}$. Since hyperbolicity is preserved under retractions, the lemma follows.

the proofs of the following two lemmas are straightforward and we left them to the reader.

LEMmA 3.4. Suppose that a group $G$ is weakly hyperbolic relative to $\left\{A, B, C_{1}, \ldots, C_{m}\right\}$, where $A$ and $B$ are conjugate subgroups of $G$. Then $G$ is weakly hyperbolic relative to $\left\{A, C_{1}, \ldots, C_{m}\right\}$.

LEMmA 3.5. Suppose that a group $G$ is weakly hyperbolic relative to $\left\{A, C_{1}, \ldots, C_{m}\right\}$ and $A$ is conjugate to a subgroup $B$ of $G$. Then $G$ is weakly hyperbolic relative to $\left\{B, C_{1}, \ldots, C_{m}\right\}$.

Now we are ready to prove Theorems 1.2 and 1.3 


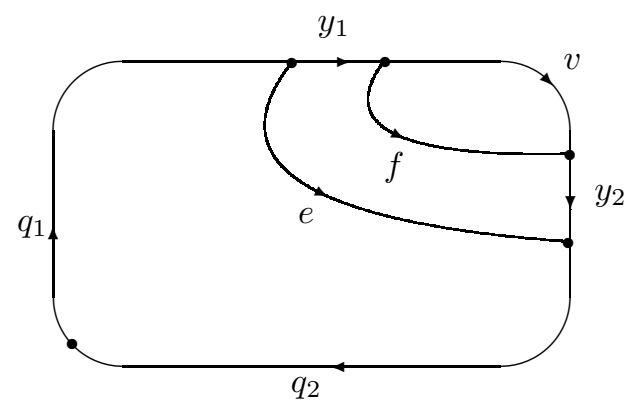

Figure 1. The decomposition of the cycle $c$ in the proof of the second assertion of Theorem 1.2 .

Proof of Theorem 1.2. The group $G$ acts on the Bass-Serre tree $T$. The vertex set $V(T)$ of $T$ is the set of the left cosets $\{g H, g \in G, i=1, \ldots, m\}$ and $G$ acts on $V(T)$ by left multiplication 23. Therefore, by Lemma 3.1 the left coset graph $\widetilde{\Gamma}$ of $G$ with respect to $H$ is quasi-isometric to $T$. Thus $G$ is weakly hyperbolic relative to $H$ by Lemma 2.4.

Let us prove the second assertion of the theorem. By Lemma 3.4 it suffices to show that $G$ is weakly hyperbolic relative to $\{A, B\}$. Let

$$
G=\left\langle H, t \mid t^{-1} a t=\mu(a)\right\rangle,
$$

where $\mu: A \rightarrow B$ is the isomorphism. By our assumption $H$ is generated by a finite set $Y$ relative to $\{A, B\}$. We put $X=\{t\} \cup Y$. Obviously $G$ is generated by the finite set $X$ relative to $A$. Let us consider the relative Cayley graphs $\Gamma_{X}^{\text {rel }}(G)$ of $G$ with respect to $\{A, B\}$ and $\Gamma_{Y}^{r e l}(H)$ of $H$ with respect to $\{A, B\}$. We can think of $\Gamma_{Y}^{r e l}(H)$ as a subgraph of $\Gamma_{X}^{r e l}(G)$. Let $M, L$ be positive constants such that $\Gamma_{Y}^{r e l}(H)$ satisfies the second condition of Proposition 2.3 Without loss of generality, we may assume $M \geq 4$. For words $U, V$ in the alphabet $X$ we denote by $\|U\|$ the length of $U$ and write $U \equiv V$ to express letter-for-letter equality of $U$ and $V$.

Consider a cycle $c$ in $\Gamma_{X}^{r e l}(G)$. We are going to check the second condition of the Proposition 3.2 Let $W$ be the label of $c$, which is a word in the alphabet $X=\{t\} \cup Y$. Suppose that $t^{ \pm 1}$ appears in $W n$ times. We want to show that $c$ admits a decomposition of type (3) with

$$
k \leq L l(c)+n \leq(L+1) l(c)
$$

terms.

If $n=0$, this is trivial since $W$ represents 1 in $H$. Further suppose that $n>0$. By the Britton Lemma on HNN-extensions (see 15), this means that $W$ has a subword of type $t^{-1} V t$ of $W$, where $V$ represents an element $a \in A$, or a subword $t U t^{-1}$, where $U$ represents an element of $B$. We consider the first case, the second one is analogous. Let $W \equiv W_{1} t^{-1} V t W_{2}$ and let $c=q_{1} y_{1} v y_{2} q_{2}$ be the corresponding decomposition of $c$, where $q_{1}, y_{1}, r, y_{2}$ and $q_{2}$ have labels $W_{1}, t^{-1}, R, t$, and $W_{2}$ respectively. Note that $t^{-1} V t$ represents some element $b$ of $B$. Thus we have

$$
[c]=\left[q_{1} e q_{2}\right]+\left[e^{-1} y_{1} f y_{2}\right]+\left[f^{-1} v\right],
$$

where $e$ and $f$ are edges of $\Gamma_{X}^{r e l}(G)$ having labels $a$ and $b$ respectively (see Figure $1)$. Note that $l\left(q_{1} e q_{2}\right)+l\left(b^{-1} v\right)=l(c)$. By the inductive assumption, $\left[q_{1} e q_{2}\right]$ and 
$\left[f^{-1} v\right]$ admit decompositions of type (3) with at most $L l\left(q_{1} e q_{2}\right)+n_{1}$ and at most $L l\left(f^{-1} v\right)+n_{2}$ terms respectively, where $n_{1}+n_{2}+2=n$. Together with (4) this gives a decomposition of type (33) for $[c]$ with at most

$$
k \leq L l\left(q_{1} a\right)+n_{1}+1+L l\left(f^{-1} r\right)+n_{2}<L l(c)+n
$$

terms. Thus $G$ is weakly hyperbolic relative to $\{A, B\}$ by Proposition 2.3

Proof of Theorem 1.3. The proof of the first assertion is analogous to that of the first assertion of Theorem 1.2 Further, recall that the amalgamated product $G=H *_{A=B} K$ is a retract of the $\mathrm{HNN}$-extension of $H * K$ with associated subgroups $A$ and $B[15$. Obviously $H * K$ is weakly hyperbolic relative to $\{A, B\}$. Thus Theorem 1.3 follows from the previous one.

Proof of Corollary 1.2. Let $F$ be a non-cyclic finitely generated free group. Then the HNN-extension

$$
G=\left\langle F, t \mid t^{-1} f t=f, f \in[F, F]\right\rangle
$$

is not finitely presented since $[F, F]$ is not finitely generated. This follows, for example, from the exactness of the Mayer-Vietoris sequence

$$
\ldots \rightarrow H_{2}(G, \mathbb{Z}) \rightarrow H_{1}([F, F], \mathbb{Z}) \rightarrow H_{1}(F, \mathbb{Z}) \rightarrow \ldots
$$

or can be proved directly by using the normal form theorem for HNN-extensions. On the other hand, $G$ is hyperbolic relative to $F$ by Theorem 1.2

Proof of Corollary 1.3. Recall a result of Rips 22. For any finitely presented group $Q$ there exists a short exact sequence

$$
1 \rightarrow N \rightarrow H \rightarrow Q \rightarrow 1,
$$

where $H$ is a finitely generated hyperbolic group and $N$ is a normal subgroup of $H$ generated by 2 elements $a, b$. Let $Q$ be a group with undecidable word problem. Then obviously the membership problem for $N$ (that is, given an element $h \in H$, to decide whether $h \in N$ ) is undecidable. Consider the HNN-extension

$$
G=\left\langle H, t \mid t^{-1} a t=a, t^{-1} b t=b\right\rangle,
$$

which is a finitely presented metahyperbolic group. Notice that $[t, h]=1$ for $h \in H$ if and only if $h \in N$. Therefore, the word problem is undecidable in $G$.

Proof of Corollary 1.4. In 8 , Burger and Mozes showed that there exists an infinite simple group $G$ which is an amalgamated product of two finitely generated free groups along finitely generated subgroups. Evidently any such a group $G$ is metahyperbolic by Theorem 1.3 since the free group is weakly hyperbolic with respect to any finitely generated subgroup. (The fact is almost trivial for free groups; in more general settings it can be found in 11.)

\section{References}

[1] E. Alibegovic, A Combination Theorem for Relatively Hyperbolic Groups, prep., 2003, available at http://arxiv.org/abs/math.GR/0310257

[2] M. Bestvina, M. Feighn, A combination theorem for negatively curved groups, J. Diff. Geom., 35 (1992), 85-101.

[3] B.H. Bowditch, Relatively hyperbolic groups, prep., 1999.

[4] B.H. Bowditch, Intersection numbers and hyperbolicity of the curve complex, prep., 2003 
[5] S.G. Brick, J.M. Corson, On Dehn functions of amalgamations and strongly undistorted subgroups, Int. J. Alg. Comp., 10 (2000), no. 5, 665-681.

[6] M. Bridson, A. Haefliger, Metric spaces of non-positive curvature, Springer, 1999.

[7] I. Bumagina, Conjugacy problem for relatively hyperbolic groups, submitted to Alg. Geom. Topology.

[8] M. Burger, Sh. Mozes, Finitely presented simple groups and products of trees, C. R. Acad. Sci. Paris Ser. I Math. 324 (1997), no. 7, 747-752.

[9] F. Dahmani, Combination of convergence groups, prep., 2002.

[10] B. Farb, Relatively hyperbolic groups, GAFA, 8 (1998), 810-840.

[11] S.M. Gersten, Subgroups of word hyperbolic groups in dimension 2, J. London Math. Soc., 54 (1996), 261-283.

[12] R. Gitik, On the combination theorem for negatively curved groups, Internat. J. Algebra Comput. 7 (1997), no. 2, 267-276.

[13] M. Gromov, Hyperbolic groups, Essays in Group Theory, MSRI Series, Vol.8, (S.M. Gersten, ed.), Springer, 1987, 75-263.

[14] O. Kharlampovich, A. Miasnikov, Hyperbolic groups and free constructions, Trans. Amer. Math. Soc., 350 (1998), no. 8, 571-613.

[15] R.C. Lyndon, P.E. Shupp, Combinatorial Group Theory, Springer-Verlag, 1977.

[16] I.G. Lysenok, On some algoritmic properties of hyperbolic groups, Math. USSR Izv., 35 (1990), 145-163.

[17] K.V. Mikhajlovskii, A.Yu. Ol'shanskii, Some constructions relating to hyperbolic groups, London Math. Soc. Lecture Notes Ser., 252 (1998), 263-290.

[18] A.Yu. Olshanskii, On residualing homomorphisms and $G$-subgroups of hyperbolic groups, Int. J. Alg. Comp., 3 (1993), 4, 365-409.

[19] D.V. Osin, Relatively hyperbolic groups: Intrinsic geometry, algebraic properties, and algorithmic problems, prep., 2003.

[20] D.V. Osin, Relatively hyperbolic groups and embedding theorems, prep., 2004.

[21] D.Y. Rebbechi, Algorithmic Properties of Relatively Hyperbolic Group, available at http://arxiv.org/abs/math.GR/0302245

[22] E. Rips, Subgroups of small cancellation groups, Bull. London Math. Soc., 14 (1982), no. 1, $45-47$.

[23] J-P. Serre, Trees, Springer-Verlag, 1980, Translation of "Arbres, Amalgames, $S L_{2}$ ", Astérisque, 461977.

[24] A. Szczepański, Relatively hyperbolic groups, Michigan Math. J. 45 (1998), 3, 611-618.

Department of Mathematics, 1326 Stevenson Center, Vanderbilt University, NASHVILLE TN 37240-0001, USA

E-mail address: denis.ossine@math.vanderbilt.edu, denis.osin@mtu-net.ru 